\section{$\underset{\substack{\text { hommes } \\ \text { \& migrations }}}{ }$}

\section{Hommes \& migrations}

Revue française de référence sur les dynamiques

migratoires

$1304 \mid 2013$

Frontières

\title{
Frontières territoriales et idéologiques
}

Un siècle d'affiches politiques en Suisse (1918-2013)

\section{Christelle Maire et Francesco Garufo}

\section{(2) OpenEdition}

1 Journals

\section{Édition électronique}

URL : http://journals.openedition.org/hommesmigrations/2658

DOI : 10.4000/hommesmigrations.2658

ISSN : 2262-3353

Éditeur

Musée national de l'histoire de l'immigration

\section{Édition imprimée}

Date de publication : 1 octobre 2013

Pagination : 127-133

ISBN : 978-2-919040-24-7

ISSN : 1142-852X

Référence électronique

Christelle Maire et Francesco Garufo, "Frontières territoriales et idéologiques », Hommes \& migrations [En ligne], 1304 | 2013, mis en ligne le 01 janvier 2017, consulté le 19 avril 2019. URL : http://

journals.openedition.org/hommesmigrations/2658; DOI : 10.4000/hommesmigrations.2658 
Affiche contre la révision de la loi fédérale

sur les étrangers, 1987.

( ) D. R. - Bibliothèque nationale suisse, Berne. 


\title{
FRONTIĖRES TERRITORIALES ET IDÉOLOGIQUES UN SIĖCLE D'AFFICHES POLITIQUES EN SUISSE (1918-2013)
}

par CHRISTELLE MAIRE, doctorante, sociologue, Forum suisse pour l'étude des migrations, université de Neuchâtel, FRANCESCO GARUFO, maître-assistant, Institut d'histoire, université de Neuchâtel.

\begin{abstract}
En Suisse, les représentations de l'Autre comme menace sont récurrentes dans le débat politique. Depuis la fin du XIXe siècle jusqu'aux polémiques récentes sur la construction de minarets, la place de l'étranger ne cesse de faire question. Les frontières de la Confédération helvétique sont régulièrement mises en avant pour symboliser la nécessité d'intégrer les étrangers ou, au contraire, la tentation du repli sur soi. L'iconographie de la frontière témoigne de sa double dimension paradoxale à la fois d'entrée et de mur.
\end{abstract}

\section{La frontière au cœur de l'identité suisse}

La frontière est au centre de la conception des nations, elle est l'un des marqueurs les plus évidents de son identité. En Suisse, la notion de préservation du territoire est au cœur des mythes fondateurs de l'identité nationale. La Suisse s'est toujours définie comme un exceptionnalisme, comme une nation peuplée de gens prêts à se battre pour leur indépendance. Elle construit ainsi son identité sur l'idée d'un "Sonderfall suisse qui, bien quiil ne repose que sur un fondement scientifique très mince, assure cependant des fonctions importantes dans le système des représentations collectives helvétiques ${ }^{1 "}$.

La plupart des mythes fondateurs, tels que ceux de Guillaume Tell ou d'Arnold Winkelried, mettent effectivement en scène des héros légendaires en lutte face à l'oppresseur étranger. Le combat des pères fondateurs pour établir et maintenir les frontières du pays affirme la construction d'une identité en opposition à l'étranger. Ces épisodes illustrent également le fait que le territoire a été âprement gagné et que sa défense est essentielle, tant du point de vue géographique qu'identitaire. Exaltés par les associations patriotiques ou dans le cadre de manifestations telles que les compétitions de tir ou de lutte fédérale, ces événements mythiques ont servi, dès le XIX siècle, au développement du sentiment d'appartenance et contribué à la formation d'une identité nationale commune. 
Ce processus s'inscrit dans la perspective plus large de l'essor des nationalismes européens à cette période. L'identité helvétique se traduit donc par une défense farouche des prérogatives nationales, qui prévaut encore aujourd'hui, notamment lorsqu'il s'agit de la relation de la Suisse à des instances supranationales telles que l'Union européenne.

Cette construction identitaire a contribué à la définition d'un "soi" homogène, réunifiant les parties linguistiquement et religieusement hétérogènes de la Suisse. La souveraineté et l'identité nationales demeurent des thématiques centrales des débats politiques en Suisse. Elles sont notamment le cheval de bataille du premier parti national, l'Union démocratique du centre (UDC), ce qui renforce la politisation des questions identitaires. C'est pourquoi la notion de frontière est toujours primordiale dans l'imaginaire politique suisse. Si la préservation des limites territoriales du pays a, au cours du temps, fait

Le combat des pères fondateurs pour établir et maintenir les frontières du pays affirme la construction d'une identité en opposition à l'étranger.

explicite dans la communication politique. Il est également important de noter que les usages politiques de l'identité nationale ont souvent pour effet la stigmatisation des étrangers" ${ }^{2}$ car "l'esquisse d'une image de l'étranger est au moins aussi importante dans la construction d'une identité collective que la poursuite des formes de représentations $d u$ soi ${ }^{3}$. L'image de la frontière est également utilisée par les défenseurs d'une nation plus ouverte, qui en font un symbole de l'enfermement, du repli sur soi et de l'étroitesse d'esprit de certaines proposi- tions politiques. D'un bord à l'autre de l'échiquier politique, la frontière est ainsi toujours convoquée pour penser le rapport de la Suisse et de ses habitants au monde extérieur.

\section{Construction et instrumentalisation de l'altérité}

Les définitions de la frontière, de l'"ego" et de 1'“alter" se génèrent dans et par la communication symbolique $e^{4}$, ce qui nous amène à nous pencher sur les médias politiques et à affirmer, dans une perspective identique à celle de Ruth Wodak, que les "identités nationales, en tant que formes particulières d'identités sociales, sont produites et reproduites, ainsi que transformées et démantelées discursivement ${ }^{5}$.

Dans les lignes qui suivent, la représentation de la frontière est illustrée par sept affiches couvrant près d'un siècle. Cet échantillon permet de retranscrire la manière dont les frontières sont employées pour délimiter symboliquement le "nous" et les "autres", le "Suisse" et l'étranger" dans la communication politique. Les images ainsi construites sont des outils particulièrement pertinents puisqu'elles peuvent être "envisagées comme des sources des processus et structures politiques, (...) donnant accès à une compréhension qualitative des motifs de reproduction et de communication au sein des sphères politique et culturelle ${ }^{6 "}$.

Les affiches politiques sont un outil idéal pour cerner l'intensité des débats partisans car elles cristallisent les représentations en jeu à leur paroxysme. Elles sont le reflet le plus synthétique des images et des mots du discours idéologique. En Suisse, elles sont très fréquemment exploitées, le système de la démocratie directe impliquant un

2. Gérard Noiriel, À quoi sert l'“identité nationale”?, Marseille, Saint-Denis, Agone/Comité de vigilance face aux usages publics de l'histoire (CVUH), 2007. 3. Musée national suisse (Zürich), Die Erfindung der Schweiz 1848-1998. Bildentwürfe einer Nation, Zürich, Schweizerisches Landesmuseum Bundesamt für Kultur, Chronos, 1998,

4. Ivana Marková (dir.), Dialogicité et représentations sociales, Paris, PUF, 2007, p. 7.

5. Ruth Wodak, "The discursive construction of national identities", in Discourse and Society, vol. 10, n², $1999, \mathrm{p} .4$.

6. Marion G. Müller, "Politologie und Ikonologie. Visuelle Interpretation als Politologisches Verfahren”, in Birgit Schwelling (dir.), Politikwissenschaft als Kulturwissenschaft. Theorien, Methoden, Problemstellungen, Wiesenbad, VS Verlag für Sozialwissenschaften, 2004, p. 335 (Ndlr : traduction des auteurs). 


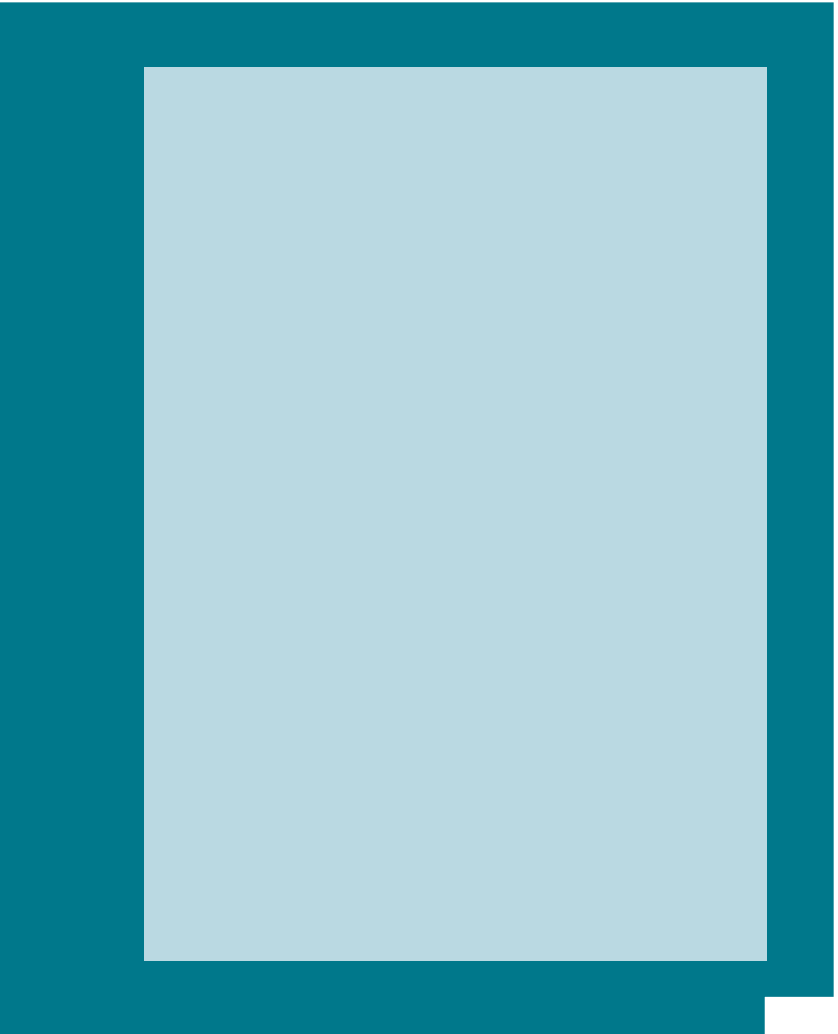

"Oui à la libre circulation des personnes, davantage de droits, moins de frontières", Genève, Syndicat interprofessionnel des travailleuses et travailleurs (SIT), 2005. () D. R.

nombre important d'échéances populaires. Les citoyens sont de ce fait appelés aux urnes trois à quatre fois par année pour se prononcer sur les objets les plus divers. Chacune de ces votations est l'occasion pour les différentes formations politiques de se confronter rhétoriquement par le biais de leurs affiches.

En Suisse, l'affiche illustrée ne trouve pleinement sa place qu'après la Première Guerre mondiale. Elle gagne en importance à partir de cette date et ne cesse de développer et d'alimenter les débats les plus polémiques au cours des décennies suivantes. Alors que l'éventail des outils du marketing s'est passablement diversifié au cours des dernières décennies, l'affiche politique occupe toujours une place de choix à l'heure actuelle.

Lors de la campagne sur l'interdiction de la construction des minarets en 2009, la diffusion par les initiants d'une affiche particulièrement polémique a ainsi suscité une controverse nationale poussant les autorités des plus importantes villes du pays à se prononcer sur son éventuelle interdiction (cf. affiche $n^{\circ} 7$ ).

L'image, notamment reprise par le Front national et le British National Party, montre une Suisse "occupée " par de nombreux minarets. L'émotion véhiculée par l'affiche politique est une composante qui marque profondément la réalité sociale helvétique. La majeure partie des affiches présentées icifont partie de l'exposition L'étranger à l'affiche $e^{7}$, qui aborde la question de la représentation de l'altérité dans la communication visuelle politique. Cette manifestation a été victime d'actes de vandalisme répétés dès sa mise en place dans l'espace public, ce qui témoigne de la sensibilité de la société helvétique aux représentations de son identité.

\section{L'entre-deux-guerres : menaces idéologiques et économiques}

La fin de la Première Guerre mondiale est marquée en Suisse par d'importants troubles sociaux, dont la grève générale de novembre 1918. Cet événement, présenté comme une tentative révolutionnaire ${ }^{8}$, traumatise le pays en raison de son caractère unique et alimente la peur du communisme. Il renforce la représentation, en vogue à partir de la fin du XIX siècle, de létranger comme vecteur d'idéologies révolutionnaires. Dans ce contexte social tendu, le débat sur "la question des étrangers", présent 
dans le pays dès les années $1880^{\circ}$, se durcit. La première attaque contre les étrangers par le biais de la démocratie directe est lancée dès septembre 1918 par la pétition "La Suisse aux Suisses", qui demande l'expulsion des étrangers "indésirables". Notre première affiche est produite en 1919 dans le cadre de cette pétition. Elle représente un Suisse aux traits stéréotypés faisant référence aux habitants des cantons de Suisse centrale, fondateurs de la Confédération. Le personnage peut être rapproché de Guillaume Tell, représentant par excellence de la résistance face à l'étranger. Les périls auxquels doit faire face la Suisse sont désormais incarnés par le communisme, une idéologie "importée" qui menace l'intégrité du pays et de ses habitants. Ses conséquences sont illustrées dans la partie inférieure gauche de l'affiche, où l'on distingue une ville en feu. Le personnage principal lutte contre une créature bicéphale pourvue de serres crochues et dont les têtes crachent une fumée noire. Le territoire est symbolisé par le drapeau national.

La menace est ici avant tout d'ordre idéologique. des minarets", Goal (Zurich), 2009 L'Autre, le non-Suisse, est le bolchevique, ce qui autorise le recours au slogan "La Suisse aux Suisses", le communiste ne pouvant en aucun cas être un Suisse véritable. Le communisme permet donc de définir, par opposition, l'identité nationale. Farouchement défendue par "Guillaume Tell”, elle est fondée sur le travail et la liberté, représentés par le personnage labourant un champ. La frontière est par conséquent tant territoriale qu'idéologique, comme l'exprime l'opposition entre les valeurs suisses et le bolchevisme. Une première catégorie de discours peut ainsi être mise en évidence : il s'agit de la défense "spirituelle" contre l'invasion étrangère. L'Autre, bolchevique en l'occurrence, corrompt l'esprit suisse et ses valeurs. Une deuxième catégorie de représentations fait référence à la menace

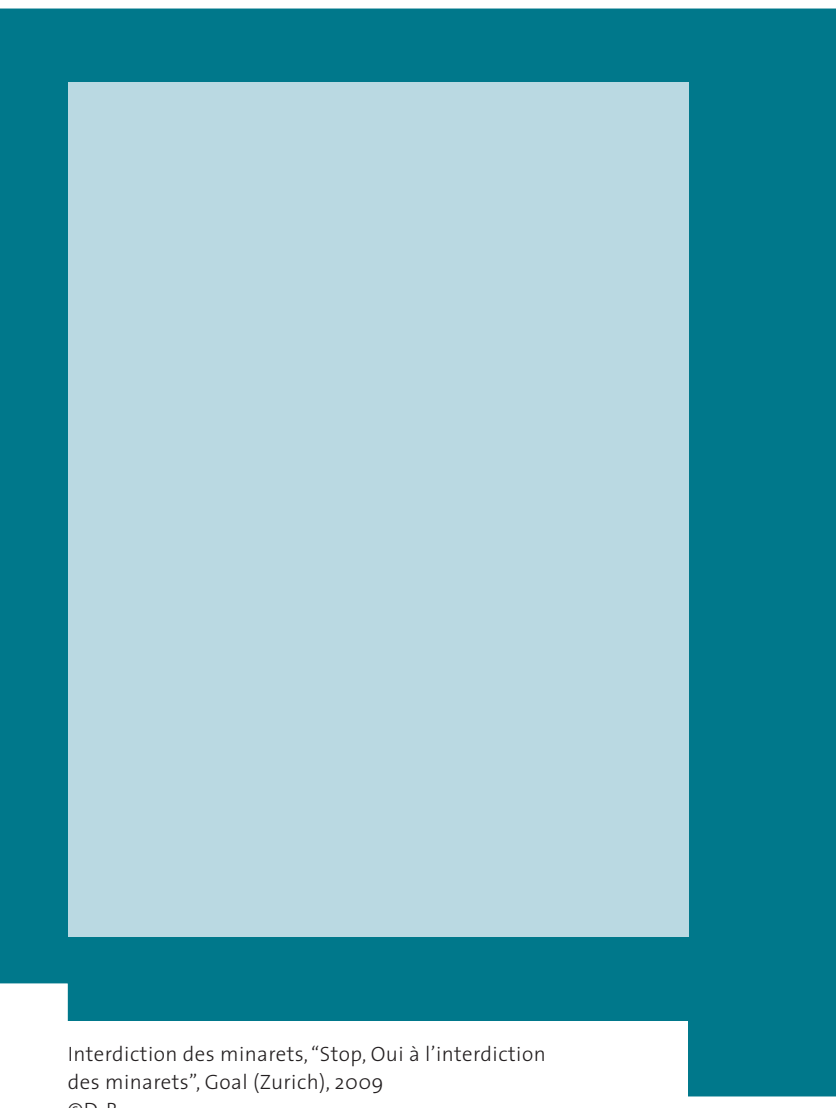

économique que constituent les étrangers. Elle vise en particulier les autorités publiques, accusées de favoriser les étrangers au détriment des Suisses. Elle est très clairement mise en image dans cette affiche de 1922, émise durant la crise économique qui met à mal les finances publiques du pays au début des années $1920^{10}$. La scène se déroule dans la ville de Bâle, dont les frontières séparent la Suisse de l'Allemagne et de la France, réunies sous le terme d'“Ausland" ("étranger"). La frontière est ici au centre de la représentation. Elle permet non seulement d'indiquer le lieu, mais surtout de jouer sur l'opposition entre le traitement accordé aux étrangers en Suisse et celui réservé aux citoyens suisses à l'étranger. Ces derniers, reconnaissables au dra-

9. Gérald Arlettaz, "La Suisse, une terre d'accueil en question : l'importance de la Première Guerre mondiale", in École française de Rome, L'Émigration politique en Europe auX XIXe et XXe siècles, Rome, École française de Rome, 1991, pp. 139-159. 10. Patrick Halbeisen, Margrit Müller, Béatrice Veyrassat (dir.), Wirtschaftsgeschichte der Schweiz im 20. Jahrhundert, Bâle, Schwabe, 2012, p. 133. 
peau sur le bras de l'un des personnages et aux vêtements traditionnels de son acolyte, sont en effet chassés sans ménagement de leurs maisons. Outre son rôle de marqueur territorial, la frontière est par conséquent utilisée pour souligner une dissymétrie de traitement. De leur côté, les étrangers du monde entier affluent vers la Suisse, dont la frontière ne bénéficie d'aucun contrôle. La barrière est ouverte à cette invasion, dont le but véritable est de profiter des largesses helvétiques. Ce sont ainsi des étrangers proches et lointains qui accourent vers la Suisse où l'argent leur est distribué du haut de la Spalentor, symbole de Bâle. L'affiche en appelle par conséquent à limiter le soutien financier accordé aux étrangers. Le thème de l'"étranger profiteur" reste encore aujourd'hui un lieu commun de la rhétorique de la droite dure.

\section{Les années 1960-1970 et la "surchauffe" économique}

Le renversement conjoncturel de l'après-Seconde Guerre mondiale entraîne en Suisse une forte et rapide augmentation de l'immigration, principalement originaire d'Italie. Dès le début des années 1960, dans le cadre d'importants débats sur la "surchauffe économique" (inflation et crise des infrastructures, en particulier du logement), diverses voix s'élèvent, à droite comme à gauche, pour demander la limitation de l'immigration.

Entre 1965 et 1974, cinq initiatives populaires "contre la surpopulation étrangère" sont lancées par les partis d'extrême droite ${ }^{11}$. C'est dans ce contexte qu'est émise l'affiche du Parti démocratique de Zurich (affiche $n^{\circ} 3$ ), qui fait référence à la venue en Suisse de travailleurs du sud de l'Europe. Le personnage, affublé des principaux stéréotypes physiques de l'immigré italien, porte une lourde valise qui symbolise son futur établissement en Suisse. Les deux représentations précédentes de la frontière sont employées conjointement. Le drapeau évoque le territoire lui-même, tandis que les barrières douanières sont représentées par un treillis métallique. Les promoteurs de l'initiative expriment ainsi leur volonté de freiner l'“invasion étrangère". C'est dans le cadre de ces consultations qu'apparaissent les premières affiches favorables à l'immigration. Les positions varient entre la gauche, qui exprime sa solidarité envers les camarades étrangers, et la droite libérale, qui met en avant l'importance du rôle économique de l'immigration et la tradition d'ouverture de la Suisse. À travers une représentation évanescente du pays, l'affiche $n^{\circ} 4$ exprime ainsi le rejet d'une initiative qui aurait pour effet de diluer les valeurs nationales qu'entend défendre le Parti radical suisse. Après le drapeau et les frontières, les contours du territoire illustrent un troisième type de représentation du pays et de ses limites, déjà présent dans l'entre-deux-guerres.

\section{Les trente dernières années : entre asile et circulation des personnes}

Le déclin du Mouvement contre la surpopulation étrangère dès le milieu des années 1970 marque un renouveau dans les discours adressés contre les étrangers. Alors que, jusque-là, les travailleurs du sud de l'Europe étaient la cible principale des protestations, les requérants d'asile extra-européens La frontière est ici perçue et des Balkans deviennent le nouveau bouc émissaire de la droite dure. Ce tournant idéo-logique et argumentatif se manifeste dans l'établissecomme une restriction à la liberté des travailleurs et comme une entrave au développement des droits sociaux dans le pays. ment de nouvelles figures rhétoriques telles que celle du criminel étranger et du faux réfugié. Les bouleversements politiques et sociaux du contexte migratoire, notamment l'intensification des flux de l'asile vers l'Europe dans les années 1990, puis l'entrée en vigueur de l'accord sur la libre circulation des personnes en 2000 
et l'adhésion de la Suisse à l'espace Schengen en 2005, ont fortement contribué au renouvellement de la perception de la frontière et de la peur de l'invasion. Depuis deux décennies maintenant, les thématiques de l'asile et de l'Union européenne sont au cour de la façon dont la Suisse pense sa relation à l'Autre et établit les frontières de son identité.

En exploitant la frontière comme symbole de la xénophobie et du repli sur soi, les deux affiches suivantes illustrent l'opposition de certaines formations politiques aux représentations véhiculées par les chantres de la fermeture du pays. Sur la première, éditée par le comité référendaire contre la révision de la loi sur l'asile et le séjour des étrangers de 1987, l'image occupant la totalité de l'espace montre en quatre dessins le processus d'une croix blanche se repliant sur elle-même et se transformant en un cube formé de briques blanches. Les quatre figures apparaissent sur un fond rouge, qui s'assombrit progressivement. La croix helvétique disparaît peu à peu pour laisser la place à un mur, qui symbolise la fermeture progressive des frontières engendrée par l'acceptation de ces deux révisions. Elle fait également référence à la figure de la forteresse régulièrement utilisée dans les discours sur l'immigration en Europe.

L'affiche dénonce par conséquent les restrictions de l'asile et du droit des étrangers proposées dans le cadre de cette votation et les conséquences néfastes (cf. l'assombrissement de la couleur de fond) sur la tradition d'accueil helvétique, également considérée comme constitutive de l'identité nationale du pays. Ici, le mur-frontière vient symboliser cette fermeture juridique et idéologique du pays.

Dans l'affiche suivante, produite en 2005 dans le cadre de la campagne pour l'extension des accords bilatéraux, la frontière est également perçue comme un élément entravant les droits de certaines personnes. Les limites territoriales sont survolées par une nuée de personnages affublés d'ailes de papier sur lesquelles on peut distinguer des inti- tulés tels que "contrat de travail", "convention collective" ou "salaire minimum". Ces personnages portent des attributs physiques, vestimentaires et sociaux très diversifiés. Au sol, trois hommes portant les marques classiques de la bourgeoisie et du patronat (costume trois-pièces, haut-de-forme, cigare) manifestent leur mécontentement, menaçant de leurs poings la foule qui survole librement ces barrières.

La frontière est ici perçue comme une restriction à la liberté des travailleurs et comme une entrave au développement des droits sociaux dans le pays. Elle est donc un risque pour la prospérité économique et symbolise l'emprise du patronat sur le marché de l'emploi. La vision de la Suisse véhiculée par cette affiche l'inscrit dans une perspective proche de celle que défend le Parti socialiste, favorable aux accords de Schengen et Dublin en opposition à l'UDC pour qui la "suppression des frontières" est synonyme d'insécurité, de chômage et d'immigration massive.

\section{La frontière, notion essentielle de l'imaginaire politique suisse}

Les sept affiches analysées témoignent de la permanence de la frontière dans la culture visuelle politique suisse. À la fin de la Première Guerre mondiale, la préoccupation principale est celle de la "défense nationale spirituelle", qui s'exprime notamment par un anticommunisme virulent. Les difficultés économiques que traverse la Suisse dès 1920 favorisent également l'émergence de la figure de l'"étranger profiteur". Les représentations de la Suisse et de ses frontières se déclinent principalement de trois manières (drapeau, barrière douanière et contour du territoire), auxquelles viennent s'ajouter des représentations métaphoriques ${ }^{12}$. Les contextes de leur émission et les sujets en votation déterminent, d'une part, le recours à certaines figures typiques et, d'autre part, l'identification d'un péril changeant, que l'on pourrait qualifier 


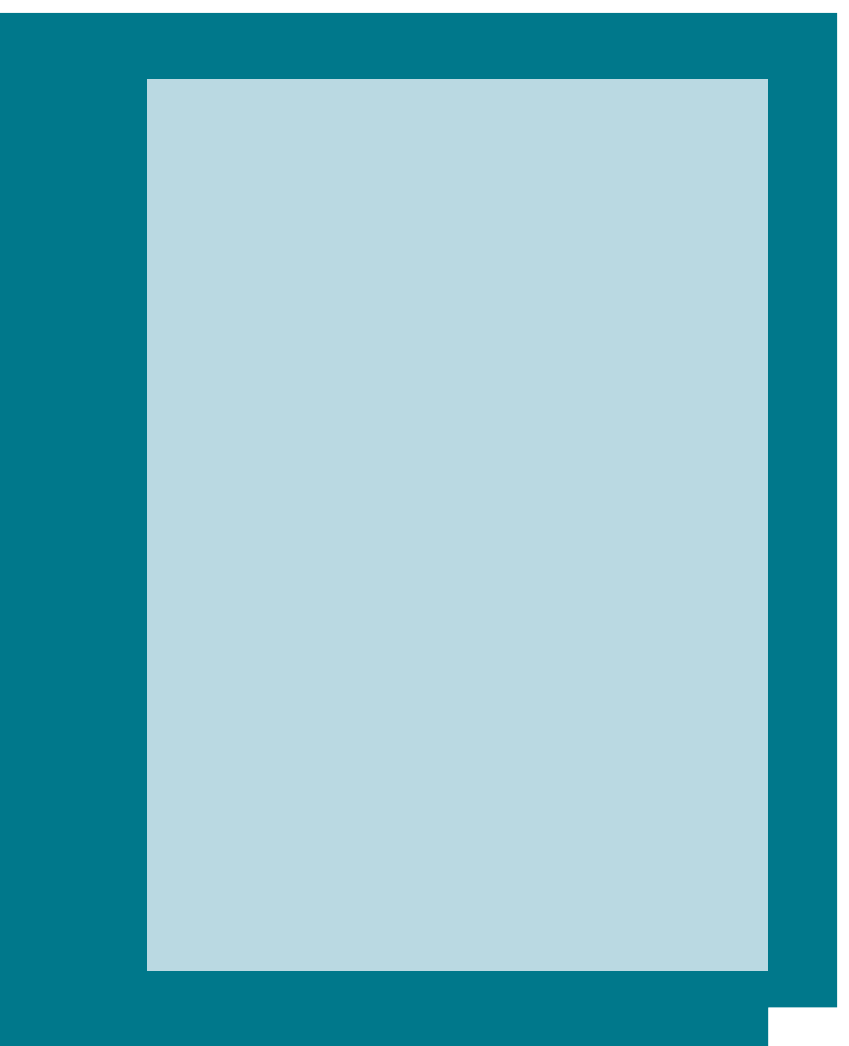

"Stop à la surpopulation étrangère, Parti Démocratique, Liste 7", Atelier 8703 (Erlenbach), 1966

C) Bibliothėoue nationale suisse, Berne

schématiquement de sécuritaire/identitaire ou d'économique. Les bases de la représentation de l'identité et de l'altérité sont ainsi jetées dès la première phase de développement de l'affiche politique illustrée.

Les premières affiches favorables aux étrangers apparaissent pour leur part dans le cadre de la forte croissance économique des Trente Glorieuses. Elles s'appuient sur trois idées : solidarité envers les travailleurs étrangers, pour la gauche, utilité économique et tradition d'accueil, pour la droite libérale. Les années 1980 marquent l'affirmation d'un discours d'ouverture utilisant la figure de la frontière pour dénoncer le repli sur soi et la diminution de la liberté et des droits individuels. Avec pour toile de fond les négociations sur la libre circulation, la confrontation entre ouverture et fermeture des frontières reste extrêmement vive jusqu'à aujourd'hui.

La représentation des frontières semble, par conséquent, ne pas évoluer à travers le temps, tandis que les menaces qui pèsent sur le pays changent au gré des contextes politiques, économiques et sociaux d'émission des affiches.

Néanmoins, au-delà de la simple préservation physique du territoire, la frontière reste le symbole de l'appartenance nationale, de la limite entre ceux qui font ou ne font pas partie de la communauté suisse. Elle est le reflet de la conception de l'État-nation, de son ouverture ou de sa fermeture au multiculturalisme et à la diversification de la société helvétique. La dernière affiche de notre corpus en est certainement l'illustration la plus emblématique. En montrant une Suisse envahie par les minarets, l'Union démocratique du centre (UDC) manifeste symboliquement sa perception de l'islam comme une menace interne au pays. La femme à la burqa et les édifices religieux, qui prennent la forme de missiles, sont représentés à l'intérieur même du drapeau national, icône majeure de l'identité nationale. En procédant de la sorte, l'affiche dénonce, par la figure de la frontière violée, la présence de cultures allogènes dans le pays. Elle établit une vision de l'identité nationale qui se trouve altérée par ces éléments et place explicitement, par son slogan "Stop", les musulmans hors de sa définition de la communauté nationale. L'image de la frontière conserve ainsi toute sa fonction symbolique de marqueur identitaire. 